\title{
Finite-precision measurement does not nullify the Kochen-Specker theorem
}

\author{
Adán Cabello \\ Departamento de Física Aplicada II, Universidad de Sevilla, 41012 Sevilla, Spain
}

(Dated: October 28, 2018)

\begin{abstract}
It is proven that any hidden variable theory of the type proposed by Meyer [Phys. Rev. Lett. 83, 3751 (1999)], Kent [ibid. 83, 3755 (1999)], and Clifton and Kent [Proc. R. Soc. London, Ser. A 456, 2101 (2000)] leads to experimentally testable predictions that are in contradiction with those of quantum mechanics. Therefore, it is argued that the existence of dense Kochen-Specker-colorable sets must not be interpreted as a nullification of the physical impact of the Kochen-Specker theorem once the finite precision of real measurements is taken into account.
\end{abstract}

PACS numbers: 03.65.Ta, 03.65.Ud

\section{THE KOCHEN-SPECKER THEOREM}

The Kochen-Specker (KS) theorem [1, 2, 3 shows one of the most fundamental features of quantum mechanics (QM): measurements do not reveal preexisting values. More precisely, it asserts that any hidden variable theory that satisfies QM must be contextual (i.e., the predefined results must change depending on which other compatible measurements are performed).

Its original mathematical proof [3] is based on the observation that, for a physical system described in QM by a Hilbert space of dimension $d \geq 3$, it is possible to find a set of $n$ projection operators $P_{i}$ which represent yes-no questions about the physical system so that none of the $2^{n}$ possible sets of "yes" or "no" answers is compatible with the sum rule of QM for orthogonal resolutions of the identity (i.e., if the sum of a subset of mutually orthogonal projection operators is the identity, one and only one of the corresponding answers ought to be "yes") [4]. Yesno questions can also be represented by the vectors $\hat{v}_{i}$ onto which $P_{i}$ projects. $\hat{v}_{i}$ can be assumed to belong to $S^{d-1}$, the unit sphere in $\mathbb{R}^{d}$. If there are predefined noncontextual yes-no answers, then there will exist a function $f: S^{d-1} \longrightarrow\{0,1\}$ such that

$$
\sum_{i=1}^{d} f\left(\hat{v}_{i}\right)=1 \text { whenever } \sum_{i=1}^{d} P_{i}=\mathbb{I}
$$

where $\left\{P_{i}\right\}_{i=1}^{d}$ is a set of orthogonal projectors and II denotes the identity, $f\left(\hat{v}_{i}\right)=1$ means that the predefined answer to the yes-no question represented by $P_{i}$ is "yes," and $f\left(\hat{v}_{j}\right)=0$ means that the answer to $P_{j}$ is "no." If such a function exists for a given set of vectors, it is said that the set is "KS-colorable;" if it does not exist, then it is said that the set is "KS-uncolorable" and serves as a proof of the KS theorem. The original proof [3] consists of a KS-uncolorable set of 117 vectors in $S^{2}$. The smallest proofs currently known have 31 vectors in $S^{2}$ [5] and 18 vectors in $S^{3}[$ [6].

\footnotetext{
*Electronic address: adan@us.es
}

A simple physical interpretation of the projection operator $P_{i}$ onto $\hat{v}_{i} \in S^{2}$ can be given in terms of the spin components along $\hat{v}_{i}$ of a spin-1 particle. Using a suitable representation for $J_{x}, J_{y}$, and $J_{z}$ [7], the relation

$$
P_{i}=\mathbb{I}-\frac{J_{i}^{2}}{\hbar^{2}}
$$

defines a one-to-one correspondence between the projector $P_{i}$ onto $\hat{v}_{i}$ and the square of the spin component along $\hat{v}_{i}$, denoted by $J_{i}^{2}$. Therefore, a measurement of $P_{i}$ represents the yes-no question "does the square of the spin component along $\hat{v}_{i}$ equal zero?" The eigenvalue 1 corresponds to the answer "yes," and the degenerate eigenvalues 0 to the answer "no." The operators $J_{x}^{2}, J_{y}^{2}$, $J_{z}^{2}$ (or any other three squares of spin components along three orthogonal directions) commute, so that the corresponding observables can be measured simultaneously. In addition, since

$$
J_{x}^{2}+J_{y}^{2}+J_{z}^{2}=2 \hbar^{2} \mathbb{I}
$$

then QM predicts that the results of measuring observables $J_{x}^{2}, J_{y}^{2}, J_{z}^{2}$ must be one 0 and two $\hbar^{2}$. Analogously, the projectors $P_{x}, P_{y}, P_{z}$ commute, so that the corresponding yes-no questions can be measured simultaneously. Using Eq. (2), Eq. (3) becomes

$$
P_{x}+P_{y}+P_{z}=\mathbb{I} \text {. }
$$

Therefore, according to QM, the answers to $P_{x}, P_{y}, P_{z}$ must be one "yes" (represented by 1) and two "no" (represented by 0$)$.

The fact that a function $f: S^{2} \longrightarrow\{0,1\}$ satisfying the condition (11) does not exist means that all $P_{i}\left(J_{i}^{2}\right)$ cannot have predefined answers (values) compatible with relation (4) [(3)].

\section{PROOFS OF "NULLIFICATION"}

Godsil and Zaks [8] have shown that the threedimensional rational unit sphere $S^{2} \cap \mathbb{Q}^{3}$ can be colored using only three colors such that orthogonal vectors are differently colored. A corollary of this result has 
been recently used by Meyer [9] to show that $S^{2} \cap \mathbb{Q}^{3}$ is KS-colorable. Therefore, one can assign predefined answers if one is restricted to those $P_{i}$ which project onto $\hat{v}_{i} \in S^{2} \cap \mathbb{Q}^{3}$. The set $S^{2} \cap \mathbb{Q}^{3}$ is dense in $S^{2}$ and therefore vectors in $S^{2} \cap \mathbb{Q}^{3}$ cannot be distinguished from those in $S^{2}$ by finite-precision measurements. This leads Meyer to conclude that "finite-precision measurement nullifies the Kochen-Specker theorem" [9].

Kent 10] has shown that dense KS-colorable sets exist in any arbitrary finite-dimensional real or complex Hilbert space. This leads him to conclude that "noncontextual hidden variable (NCHV) theories cannot be excluded by theoretical arguments of the KS type once the imprecision in real world measurements is taken into account" [10]. More recently, Clifton and Kent [11] have constructed a NCHV model for any finite-dimensional Hilbert space that they claim is consistent with all the statistical predictions of QM. This allows them to conclude that "all the predictions of nonrelativistic QM that are verifiable to within any finite precision can be simulated classically by NCHV theories" [11].

In response, Ax and Kochen 12 have argued that the study of the effect of finite-precision measurements on the KS theorem requires a different formalization which is still missing. In 13 there is a criticism of the physical interpretation of the existence of KS-colorable sets. Havlicek et al. 14] have argued that any possible KS coloring of the rational unit sphere is not physically satisfactory. Mermin 15 has argued that the continuity of probabilities under slight changes in the experimental configuration weighs against the conclusions in 19, 10 . Appleby has expanded on Mermin's discussion 16] and argued that in the models of Meyer, Kent, and Clifton the very existence of an observable is contextual [17] and measurements do not reveal preexisting classical information 18.

In this paper I shall prove that any possible KS coloring of the rational unit sphere of the type proposed in [9] leads to predictions which differ from those of QM and, therefore, that any NCHV theory which assigns definite colors to the rational unit sphere can be discarded on experimental grounds even if finite-precision measurements are used. In addition, I shall prove that any possible KS coloring of a dense set of the kind proposed by Clifton and Kent 11] also leads to predictions that differ from those of QM, and therefore explicit NCHV models like those in 111. can also be discarded on experimental grounds.

Both proofs are inspired by a lesser known type of proof of the KS theorem which does not require an entire KS-uncolorable set but only one of its subsets 19 . In particular, it is based on a proof by Stairs [20] which only requires eight of the vectors of the 117-vector KSuncolorable set in [3]. This eight-vector set appears for the first time in [21]. Stairs' proof was reformulated by Clifton 22] (see also 23, 24, 25]).

The strategy of both proofs is as follows. First we show that any NCHV theory cannot assign the value 1 to certain vectors in the set which is assumed to have predefined values. We then show that the impossibility of such an assignment leads to an inequality valid for any NCHV theory but which is violated by QM.

\section{KS COLORINGS OF THE RATIONAL SPHERE ARE INCOMPATIBLE WITH QM}

Consider the following vectors of the rational unit sphere:

$$
\begin{aligned}
\hat{A}= & \left(0, c_{A},-s_{A}\right) \\
\hat{B}= & \left(c_{B}, s_{B}, 0\right) \\
\hat{C}= & \left(c_{C} c_{D}+N c_{A} s_{C} s_{D}, N s_{A} s_{C} c_{D}\right. \\
& \left.-c_{C} s_{D}+N c_{A} s_{C} c_{D}\right)
\end{aligned}
$$

where $s_{i}=\left(1-c_{i}^{2}\right)^{1 / 2}, i$ being $A, B, C$, or $D, N=$ $\left[c_{A}^{2}+\left(s_{A} c_{D}\right)^{2}\right]^{-1 / 2}$, and $\left\{c_{i}, s_{i}, N\right\} \in \mathbb{Q}$.

Lemma 1. There is no KS coloring (1) of the rational unit sphere $S^{2} \cap \mathbb{Q}^{3}$ in which $f(\hat{A})=f(\hat{B})=f(\hat{C})=1$.

Proof via a reductio ad absurdum. Consider the following additional vectors $\hat{v}_{i} \in S^{2} \cap \mathbb{Q}^{3}$ :

$$
\begin{aligned}
& \hat{v}_{1}=(1,0,0), \\
& \hat{v}_{2}=N\left(c_{A} s_{D}, s_{A} c_{D}, c_{A} c_{D}\right), \\
& \hat{v}_{3}=(0,0,1), \\
& \hat{v}_{4}=N\left(s_{A} c_{D} s_{D},-c_{A}, s_{A} c_{D}^{2}\right), \\
& \hat{v}_{5}=(0,1,0), \\
& \hat{v}_{6}=\left(c_{D}, 0,-s_{D}\right) .
\end{aligned}
$$

If $f(\hat{A})=1 \Rightarrow f\left(\hat{v}_{1}\right)=f\left(\hat{v}_{2}\right)=0$, if $f(\hat{B})=1 \Rightarrow$ $f\left(\hat{v}_{3}\right)=0$, and if $f(\hat{C})=1 \Rightarrow f\left(\hat{v}_{4}\right)=0$. In addition, if $f\left(\hat{v}_{1}\right)=f\left(\hat{v}_{3}\right)=0 \Rightarrow f\left(\hat{v}_{5}\right)=1$ and if $f\left(\hat{v}_{2}\right)=f\left(\hat{v}_{4}\right)=$ $0 \Rightarrow f\left(\hat{v}_{6}\right)=1$. However, $f\left(\hat{v}_{5}\right)=1$ is incompatible with $f\left(\hat{v}_{6}\right)=1$ since $\hat{v}_{5}$ and $\hat{v}_{6}$ are orthogonal.

Let us consider an ensemble of spin-1 particles and let us assume that any particle has a definite color (1 or 0$)$ for every vector of the rational unit sphere and in particular for $\hat{A}, \hat{B}$, and $\hat{C}$. Let $P(B)$ be the probability of finding a particle with $f(\hat{B})=1$ in such an ensemble, $P(A \wedge B \wedge C)$ be the probability of finding $f(\hat{A})=f(\hat{B})=f(\hat{C})=1, P(A \wedge B \wedge \neg C)$ be the probability of finding $f(\hat{A})=f(\hat{B})=1$ and $f(\hat{C})=0$, and $P(A \mid B)$ be the probability of finding $f(\hat{A})=1$ if $f(\hat{B})=1$. Note that such probabilities make sense in a NCHV theory but not in QM. From the point of view of a NCHV theory, $P(A \mid B)$ can be obtained by means of two alternative but equivalent methods 22, 25]: either preparing the particles in a quantum eigenstate of the square of the spin component along $\hat{B}$ with eigenvalue 0 [7], measuring the square of the spin along $\hat{A}$, and counting the number of events in which the eigenvalue 0 has been obtained, or preparing the particles in pairs in the singlet state, measuring the square of the spin along $\hat{B}$ in one of the particles and the square of the spin along $\hat{A}$ 
in the other, and counting the number of events in which both results are 0 .

Lemma 2. There is no KS coloring (11) of the rational unit sphere $S^{2} \cap \mathbb{Q}^{3}$ compatible with all the statistical predictions of QM.

Proof. The following inequality must be satisfied in any NCHV theory:

$$
P(B) \geq P(A \wedge B \wedge \neg C)+P(\neg A \wedge B \wedge C) .
$$

Lemma 1 shows that

$$
P(A \wedge B \wedge C)=0
$$

Therefore, the inequality (14) can be written as

$$
P(B) \geq P(A \wedge B)+P(B \wedge C),
$$

which is equivalent to

$$
P(B) \geq P(B) P(A \mid B)+P(B) P(C \mid B) .
$$

Inequality (17) can be simplified to

$$
1 \geq P(A \mid B)+P(C \mid B)
$$

Let us define

$$
F_{\mathrm{NCHV}}=P(A \mid B)+P(C \mid B) .
$$

Then, according to inequality (18), any NCHV theory will predict

$$
F_{\mathrm{NCHV}} \leq 1
$$

On the other hand, in QM the equivalent of Eq. (19) is

$$
F_{\mathrm{QM}}=|\langle\hat{A} \mid \hat{B}\rangle|^{2}+|\langle\hat{C} \mid \hat{B}\rangle|^{2} .
$$

In particular, if we choose

$$
\begin{aligned}
& c_{A}=\frac{104}{185} \\
& c_{B}=\frac{10209400000}{12605796209} \\
& c_{C}=\frac{490231}{789769} \\
& c_{D}=\frac{105}{137}
\end{aligned}
$$

then we obtain

$$
F_{\mathrm{QM}}=1.108
$$

which contradicts the prediction of $\mathrm{NCHV}$ theories given by inequality (20) 26].

If all the particles of the ensemble have predefined "colors" along $\hat{A}, \hat{B}$, and $\hat{C}$, then $F$ has an "exact" value for that ensemble. To check that value, we must perform tests along $\hat{A}$ and $\hat{B}$, and along $\hat{C}$ and $\hat{B}$. When we perform tests along $\hat{A}$ and $\hat{B}$, their results reveal either the real colors of $\hat{A}$ and $\hat{B}$ or the colors of $\hat{A}^{\prime}$ and $\hat{B}^{\prime}$ that are, respectively, infinitesimally close to them. In any NCHV theory in which measurements are assumed to reveal predefined colors and because of the own definition of "precision," in a higher precision test along $\hat{A}$ and $\hat{B}$, the number of results revealing the true colors of $\hat{A}$ and $\hat{B}$ is higher than in a lower precision test. Therefore, successive tests with increasing precision will give us the true colors with a higher probability. Thus, they will give us decreasing bounds around the exact value of $F$. Therefore, even experiments with finite-precision can discriminate between the prediction of NCHV theories (20) and the prediction of QM (26).

\section{CLIFTON AND KENT'S NCHV MODEL IS INCOMPATIBLE WITH QM}

Clifton and Kent's NCHV model [1] is based on the existence of dense $\mathrm{KS}$-colorable sets $\mathcal{D}$ with the remarkable property that every projector in $\mathcal{D}$ belongs to only one resolution of the identity. Moreover, the function $f$ defined over $\mathcal{D}$ and satisfying condition (11) must be "sufficiently rich to recover the statistics of any quantum state" 11]. They claim that the existence of $\mathcal{D}$ "defeats the practical possibility of falsifying NCHV on either nonstatistical or statistical grounds" 11 .

Let us outline a proof that shows that such a claim is not correct. Consider a particular $\mathcal{D}$ dense in $S^{2}$ and two vectors $\hat{A}^{\prime}, \hat{B}^{\prime} \in \mathcal{D}$. Suppose that $\hat{A}^{\prime}$ is infinitesimally close to $\frac{1}{\sqrt{3}}(1,1,1)$ and $\hat{B}^{\prime}$ is infinitesimally close to $\frac{1}{\sqrt{3}}(1,1,-1)$.

Lemma 3. Given an ensemble of systems such that each system is described by $\mathcal{D}$, the probability of finding an individual system in which a KS coloring (1) satisfies $f\left(\hat{A}^{\prime}\right)=f\left(\hat{B}^{\prime}\right)=1$ is infinitesimally close to zero if such coloring must simulate the predictions of QM.

Proof. Consider six additional vectors $\hat{v}_{i}^{\prime} \in \mathcal{D}$ such that $\hat{v}_{1}^{\prime}$ and $\hat{v}_{2}^{\prime}$ are both infinitesimally close to being orthogonal to $\hat{A}^{\prime} ; \hat{v}_{3}^{\prime}$ and $\hat{v}_{4}^{\prime}$ are both infinitesimally close to being orthogonal to $\hat{B}^{\prime} ; \hat{v}_{1}^{\prime}, \hat{v}_{3}^{\prime}$, and $\hat{v}_{5}^{\prime}$ are mutually orthogonal; $\hat{v}_{2}^{\prime}, \hat{v}_{4}^{\prime}$, and $\hat{v}_{6}^{\prime}$ are mutually orthogonal; $\hat{v}_{5}^{\prime}$ and $\hat{v}_{6}^{\prime}$ are infinitesimally close to being orthogonal [27]. The fact that eight vectors with the above properties exist in $\mathcal{D} \in S^{2}$ is not excluded in $[1]$. Since $f$ must simulate the predictions of QM, if $f\left(\widehat{A^{\prime}}\right)=1$, then the probability of $f\left(\hat{v}_{1}^{\prime}\right)=1$ must be $\left|\left\langle\hat{v}_{1}^{\prime} \mid \hat{A}^{\prime}\right\rangle\right|^{2}$, that is, infinitesimally close to zero, because $\hat{A}^{\prime}$ and $\hat{v}_{1}^{\prime}$ are infinitesimally close to being orthogonal. The same argument states that if $f\left(\hat{A}^{\prime}\right)=1$, the probability of $f\left(\hat{v}_{2}^{\prime}\right)=1$ must be infinitesimally close to zero. Therefore, if $f\left(\hat{A}^{\prime}\right)=1$, then $f\left(\hat{v}_{1}^{\prime}\right)=f\left(\hat{v}_{2}^{\prime}\right)=0$ for almost every system of an ensemble. Using the same reasoning, if $f\left(\hat{B}^{\prime}\right)=1$, then $f\left(\hat{v}_{3}^{\prime}\right)=f\left(\hat{v}_{4}^{\prime}\right)=0$ for almost every system of an ensemble. In addition, if $f\left(\hat{v}_{1}^{\prime}\right)=f\left(\hat{v}_{3}^{\prime}\right)=0 \Rightarrow f\left(\hat{v}_{5}^{\prime}\right)=1$ and if $f\left(\hat{v}_{2}^{\prime}\right)=f\left(\hat{v}_{4}^{\prime}\right)=0 \Rightarrow f\left(\hat{v}_{6}^{\prime}\right)=1$. Since $f$ must simulate 
the predictions of $\mathrm{QM}$, if $f\left(\hat{v}_{5}^{\prime}\right)=1$, then the probability of $f\left(\hat{v}_{6}^{\prime}\right)=1$ must be $\left|\left\langle\hat{v}_{6}^{\prime} \mid \hat{v}_{5}^{\prime}\right\rangle\right|^{2}$, that is, infinitesimally close to zero, because $\hat{v}_{5}^{\prime}$ and $\hat{v}_{6}^{\prime}$ are infinitesimally close to being orthogonal [28].

Lemma 4. There is no KS coloring (1) of $\mathcal{D} \in S^{2}$ compatible with all the statistical predictions of QM.

Proof. I will use the same notation used in the proof of Lemma 2. According to Lemma 3, in any NCHV theory

$$
P\left(A^{\prime} \wedge B^{\prime}\right) \approx 0
$$

However, according to QM,

$$
\left|\left\langle\hat{A}^{\prime} \mid \hat{B}^{\prime}\right\rangle\right|^{2} \approx \frac{1}{9} .
$$

\section{CONCLUSION}

The reason why dense sets in [9, 10, 11] do not lead to NCHV theories that simulate QM can be summarized as follows: most of the many possible KS colorings of these sets must be statistically irrelevant in order to reproduce some of the statistical predictions of QM. Then, the remaining statistically relevant KS colorings cannot reproduce some different statistical predictions of QM. I therefore conclude that the existence of KS-colorable sets that are dense in the corresponding Hilbert spaces, like those in [9, 10, 11], does not lead by itself to a NCHV theory capable of eluding statistical KS-type proofs, and must therefore not be interpreted as a nullification of the physical impact of the KS theorem once the finiteprecision of measurements is taken into account.

\section{Acknowledgments}

The author thanks D. M. Appleby, R. Clifton, C. A. Fuchs, G. García de Polavieja, A. Kent, A. J. López Tarrida, F. Martín Maroto, N. D. Mermin, D. A. Meyer, A. Peres, E. Santos, C. Serra, C. Simon, and M. Żukowski for discussions on this topic and acknowledges support from the organizers of the Sixth Benasque Center for Physics, the University of Seville Grant No. OGICYT191-97, the Junta de Andalucía Grant No. FQM-239, and the Spanish Ministerio de Ciencia y Tecnología Grant Nos. BFM2000-0529 and BFM2001-3943.
[1] E. P. Specker, Dialectica 14, 239 (1960).

[2] J. S. Bell, Rev. Mod. Phys. 38, 447 (1966).

[3] S. Kochen and E. P. Specker, J. Math. Mech. 17, 59 (1967).

[4] A. Peres, Quantum Theory: Concepts and Methods (Kluwer, Dordrecht, 1993).

[5] J. H. Conway and S. Kochen, reported in Ref. [4, p. 114.

[6] A. Cabello, J. M. Estebaranz, and G. García Alcaine, Phys. Lett. A 212, 183 (1996).

[7] See, for instance, A. Messiah, Mécanique Quantique (Dunod, Paris, 1964) [latest English version Quantum Mechanics (Dover, New York, 2000)], Sec. XIII-21, or Ref. [4], p. 199.

[8] C. D. Godsil and J. Zaks, University of Waterloo Research Report No. CORR 88-12, 1988 (unpublished).

[9] D. A. Meyer, Phys. Rev. Lett. 83, 3751 (1999).

[10] A. Kent, Phys. Rev. Lett. 83, 3755 (1999).

[11] R. Clifton and A. Kent, Proc. R. Soc. London, Ser. A 456, 2101 (2000).

[12] J. Ax and S. Kochen (unpublished).

[13] A. Cabello, e-print quant-ph/9911024 (unpublished).

[14] H. Havlicek, G. Krenn, J. Summhammer, and K. Svozil, J. Phys. A 34, 3071 (2001).

[15] N. D. Mermin, e-print quant-ph/9912081 (unpublished).

[16] D. M. Appleby, e-print quant-ph/0005010 (unpublished).

[17] D. M. Appleby, Phys. Rev. A 65, 022105 (2002).

[18] D. M. Appleby, e-print quant-ph/0109034 (unpublished).

[19] For a classification of the proofs of the KS theorem, see A. Cabello and G. García Alcaine, J. Phys. A 29, 1025 (1996).

[20] A. Stairs, Philos. Sci. 50, 578 (1983).

[21] S. Kochen and E. P. Specker, in Symposium on the
Theory of Models, Proceedings of the 1963 International Symposium at Berkeley, edited by J. W. Addison et al. (North-Holland, Amsterdam, 1965), p. 177; reprinted in The Logico-Algebraic Approach to Quantum Mechanics. Volume I: Historical Evolution, edited by C. A. Hooker (Reidel, Dordrecht, 1975), p. 263, and in E. P. Specker, Selecta (Birkhäuser Verlag, Basel, 1990), p. 209.

[22] R. Clifton, Am. J. Phys. 61, 443 (1993).

[23] H. Bechmann Johansen, Am. J. Phys. 62, 471 (1994).

[24] P. E. Vermaas, Am. J. Phys. 62, 658 (1994).

[25] A. Cabello and G. García Alcaine, J. Phys. A 28, 3719 (1995).

[26] If we were not restricted to rational vectors, then the maximum contradiction would occur when $F_{\mathrm{QM}}=1.172$, as can be numerically found. Moreover, if we were not restricted to rational vectors, we could choose $\hat{B}=\hat{C}$ while preserving the remaining orthogonalities and then the maximum contradiction would occur when $F_{\mathrm{QM}}=$ $\frac{1}{9}+1$ (see Refs. 20, 22, 23, 24, 25,).

[27] For instance, $\hat{v}_{1}^{\prime} \approx \frac{1}{\sqrt{2}}(0,1,-1), \hat{v}_{2}^{\prime} \approx \frac{1}{\sqrt{2}}(1,0,-1)$, $\hat{v}_{3}^{\prime} \approx \frac{1}{\sqrt{2}}(0,1,1), \hat{v}_{4}^{\prime} \approx \frac{1}{\sqrt{2}}(1,0,1), \hat{v}_{5}^{\prime} \approx(1,0,0)$, and $\hat{v}_{6}^{\prime} \approx(0,1,0)$.

[28] R. Clifton (private communication) sees no reason to accept that if $\hat{v}_{5}^{\prime}$ and $\hat{v}_{6}^{\prime}$ are infinitesimally close to being orthogonal and have predefined values, then, in any NCHV theory which simulates QM, the systems for which $f\left(\hat{v}_{5}^{\prime}\right)=f\left(\hat{v}_{6}^{\prime}\right)=1$ must constitute a statistically irrelevant subensemble of the whole ensemble of systems. He accepts that this is the case if the ensemble was prepared in the quantum state $\left|\hat{v}_{5}^{\prime}\right\rangle$. However, there is an experimental reason that supports the above assumption: from 
the point of view of a NCHV theory, even if the ensemble is prepared in an arbitrary quantum state, any joint measurement along $\hat{v}_{5}^{\prime}$ and $\hat{v}_{6}^{\prime}$ (which are as close as one desires to being compatible observables) has a nonzero probability (which increases along with the experiment's precision) to reveal their predefined values. Experimentally, the probability of "revealing" $f\left(\hat{v}_{5}^{\prime}\right)=f\left(\hat{v}_{6}^{\prime}\right)=1$ has been found to be very close to zero, even if the ensemble has not been prepared in the state $\left|\hat{v}_{5}^{\prime}\right\rangle$. 